\title{
Journal of Real-Time Image Processing: second issue of volume 9
}

\author{
Nasser Kehtarnavaz $\cdot$ Matthias F. Carlsohn
}

Published online: 4 May 2014

(c) Springer-Verlag Berlin Heidelberg 2014

The editorial board of JRTIP met in Brussels in April 2014 where they were attending the SPIE Conference on RealTime Image and Video Processing. The editorial board will have its next meeting in San Francisco in February 2015 where the same conference will take place as part of the SPIE Electronic Imaging Symposium. In the recently held editorial meeting (see Fig. 1), various items related to the status of the journal were discussed; a summary of these items is provided below.

To address the backlog of printed versions of the accepted manuscripts that appear on Online First, we are pleased to state that the number of printed pages per issue will be increased to 200 pages per issue starting in 2015 for a total page count of 800 pages annually. It is hoped that the backlog situation is eased as a result of this page count increase. We will revisit this issue in 2015 to see whether further actions would be needed to resolve this issue.

The journal performance numbers that have become available for the year 2013 clearly reflect the elevated recognition level of JRTIP. In 2013, similar to 2012, the acceptance rate of manuscripts submitted to JRTIP has remained at about $25 \%$. This is an indication that our review process is conducted in such a way that only quality papers are published in JRTIP. It is worth noting that the usage factor (UF) of JRTIP, which is the ratio of the number of paper downloads over the number of papers for a 2-year period, has increased by about $75 \%$ from 2011 to

N. Kehtarnavaz $(\square)$

University of Texas at Dallas, Richardson, USA

e-mail: kehtar@utdallas.edu

\section{F. Carlsohn}

Engineering and Consultancy for Computer Vision and Image

Communication, Bremen, Germany

e-mail: Matthias.Carlsohn@t-online.de
2012. With the substantial increase in the number of downloads to $34,000+$ in 2013 from 25,500+ in 2012, it is expected that the 2013 UF would increase significantly. We would like to particularly thank the Associate Editors and the Springer Editorial Office for the major roles they have played in increasing these numerical indicators while maintaining the quality of published papers.

Here, it is worth pointing out that the third issue in 2014 will be allocated to two special issues that are scheduled to appear together. These special issues are named "Algorithms and Architectures for Real-time Multi-Dimensional Image Processing" and "Convergence of Real-Time 3D Imaging and Optical Communications".

This regular issue of JRTIP includes six papers. The first paper by Juliet et al. presents a compound image segmentation algorithm based on the mixed raster content (MRC) model (foreground/mask/background). After segmenting a compound image into different classes, each class is transformed to the MRC model according to its properties. The foreground and the background layers are compressed using JPEG 2000 while the mask layer is compressed using JBIG2. It is shown that this MRC compression achieves a higher subjective quality and coding efficiency than the state-of-the-art compression algorithms, such as JPEG, JPEG 2000 and H.264/AVC-I.

The second paper by Bartovsky et al. introduces an additional, intra-operator level of parallelism for dilation/ erosion operations. Realized in a dedicated hardware, for rectangular structuring elements with programmable size, the implementation allows obtaining real-time performances for these operators that were previously unachievable. Low latency and memory requirements are the other main attributes of this parallelism.

The third paper by Gaceb et al. describes a document classification approach based on physical layout features 


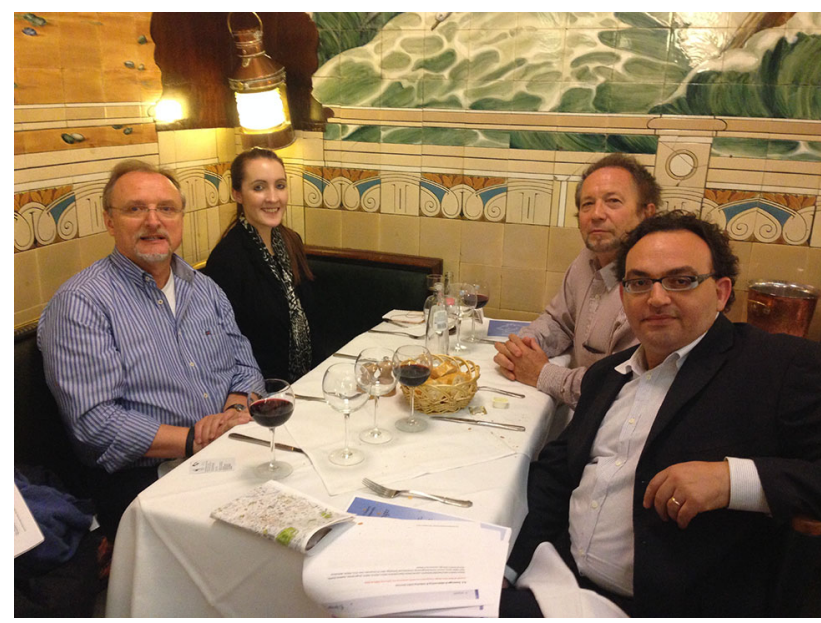

Fig. 1 Left-to-right Matthias Carlsohn, Rachel Roberts, Volodymyr Ponomaryov, Sergio Saponara

and graph b-coloring modeling. To reduce the computing time and to increase the performance, business documents are pre-classified by having an automatic recognition stage. Once a document type is identified, the corresponding information is used to improve the recognition of the logical layout, the parameterization of the system, and the sorting. The introduced method is shown to be robust to various constraints and to provide a real-time solution to the problem of business documents sorting.

The fourth paper by Kakarala et al. discusses a computationally efficient algorithm for fusing a long-exposure and a short-exposure image in the JPEG domain. The algorithm uses the spatial frequency of the discrete cosine transform to combine the uniform regions of the longexposure image with the detailed regions of the shortexposure image, thereby reducing noise while providing sharp details. Examples involving image stabilization and high-dynamic range acquisition are provided to show the effectiveness of the algorithm.

The fifth paper by Unsalan et al. covers the development of a system that can scan shiny and matte objects under ambient light using color invariant-based binary, ternary, and quaternary coded structured light-based range scanners. Using color invariants, the effect of highlights and ambient light in the scanning process is reduced, thus extracting the range data of shiny and matte objects in a robust manner. The implementation of these scanners using a TI DM6437 EVM board is reported together with a TI MSP430 microcontroller-based rotating table. It is shown that this system allows obtaining the range data of a target object from different viewpoints.

The final sixth paper by Gwosdek et al. examines electrostatic halftoning as a high-quality method for stippling, dithering, and sampling. To address the high runtime aspect of this method, the paper introduces a GPU-based Non-equispaced Fast Fourier Transform (NFFT) algorithm without special structural assumptions on the positions of nodes. For 1 million particles, this algorithm runs 50 times faster than the most efficient technique running on a CPU.

We would like to end this editorial by welcoming Gerald Schaefer as our newest member of the JRTIP editorial board. 